\title{
Estado Actual de la Formación Inicial en Competencias Básicas del Profesorado no Universitario de Colombia
}

\section{Rubén Darío Hernández Escorcia ${ }^{a}$.}

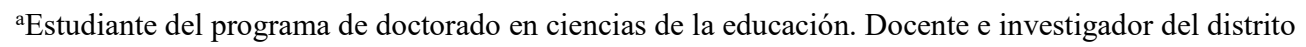
lasallista Norandino, Medellín - Colombia. ruben.hernandez@,delasalle.edu.co

\begin{abstract}
Today's society is experiencing an accelerated period of changes, a product of the development that has taken place at all levels. Education in the XXI century is not alien to this reality because it's strongly influenced by this development. Therefore, today we are seeing more clearly all the problems involved, not only the old education system and its effects on society, but fundamentally the need for training new educational agents that in their generic name are usually called, without difference, teachers, teachers or teachers. In the teacher training of recent years has played an important role the term of competence and in the case at hand is a challenge for the training of future teachers, being in charge of normal schools and universities is responsible for teacher training. From the bibliographic review of different sources it was possible to determine that the vast majority of publications found indicate the training in competencies for university teacher in practice. Being training in competencies for future teachers of incipient basic education. So this review shows us a reality of the current state of the challenge of proposing updated training plans for the initial training of non- university teaching straff.
\end{abstract}

Keywords: education, competence, training, school, university, teaching staff.

\footnotetext{
Resumen

La sociedad actual vive un periodo acelerado de cambios, producto del desarrollo que se ha dado a todos los niveles. La educación del siglo XXI no es ajena a esta realidad por estar fuertemente influenciada por dicho desarrollo. Por ello, hoy se viene apreciando con mayor nitidez toda la problemática que encierra, no sólo el viejo sistema de educación y sus efectos en la sociedad, sino fundamentalmente la necesidad de formación de nuevos agentes educativos que en su denominación genérica suelen ser llamados, sin diferencia alguna, docentes, profesores o maestros. En la formación docente de los últimos años ha jugado un papel importante el término de Competencia y en el caso que nos ocupa constituye un desafio para la formación de futuros profesores, estando a cargo de escuelas normales y universidades está responsabilidad en cuanto a formación del profesorado. A partir de la revisión bibliográfica de diferentes fuentes se pudo determinar que la gran mayoría de publicaciones encontradas señalan la formación en competencias para el profesorado universitario en ejercicio. Siendo la formación en competencias
} 
para futuros profesores de educación básica incipiente.Por lo que esta revisión nos muestra una realidad del estado actual del tema abordado y nos abre al desafio de proponer planes de formación actualizados para la formación inicial del profesorado no universitario.

Palabras clave: educación, competencias, formación, escuela, universidad, profesorado.

\section{Introducción}

La sociedad actual vive un periodo acelerado de cambios, producto del desarrollo que se ha dado a todos los niveles: tecnológicos, sociales, políticos, éticos, cienctificos, religiosos, ideológicos, educativos, etc. Todos ellos responden a los nuevos retos a los que el hombre y las sociedades en conjunto están expuestos.

La educación del siglo XXI no es ajena a esta realidad, por estar fuertemente influenciada por dicho desarrollo. Por ello, se vienen apreciando con mayor nitidez toda la problemática que encierra, no sólo el viejo sistema de educación y sus efectos en la sociedad, sino fundamentalente la necesidad de formación de nuevos docentes, profesores o maestros (Piloneta, 2014).

En la formación docente de los últimos años ha jugado un papel importante el término competencia y para la realidad que abordaremos formar al profesorado no universitario en clave competencial constituye un desafío en el país. Es así como los estudios más recientes que se reportan para Colombia hasta el momento en esta perspectiva muestran la preocupación que hay respecto a la formación en competencias para profesorado.

Por mencionar los trabajos más recientes tenemos que: Jurado Valencia (2016) en su investigación titulada "Hacia la renovación de la formación de los docentes en Colombia: ruta tradicional y ruta polivalente" propuso en una de sus fases la caratcerización de los modelos de formación de los docentes. Encontrando fisuras en el sistema educativo del país lo que no permite la cualificación del profesorado a nivel general, centrando su estudio en los docentes de universidad.

Así mismo, Restrepo, Aguirre y Gámez Navío, realizaron un estudio sobre "Las competencias del docente de maestría en universidades Colombianas" para ese mismo año. Señalando que existe una carencia en la formación competencial de los educadores en el país y que ello repercute en la calidad de la educaciòn.

Cabeza, Zapata, y Lombana (2018) realizaron el trabajo "Crisis de la profesión docente en Colombia: percepciones de aspirantes a otras profesiones" encontrando que los estudiantes que optan por estudiar otras carreras diferentes a las relacionadas con la educación, lo hacen porque ven en los programas de licenciaturas y en la carrera docente en sí, un campo poco competente y por ende muy mal pagado.

En el caso de los docentes no universitarios, que es el caso que nos ocupa la carencia o deficiencia en la formación disciplinar y competencial conduce a una docencia de mala 
calidad, con contenidos atrasados u obsoletos, con el riesgo de simplismo, superficialidad y diletantismo en lo que se enseña (Bautista y Gómez, 2017), siendo este uno de los desafíos actuales en el país en cuanto a formación del profesorado.

Escobar (2017) expresó que Colombia contaba para ese entonces con 315.971 maestros aproximadamente, para los cerca de 8 millones de estudiantes de las instituciones educativas del secor oficial. Es decir, aproximadamente un maestro por cada 25 estudiantes. Todos estos docentes han sido formados en su mayoría en las escuelas normales y las universidades.

Donde en estas últimas, la formación se lleva a cabo en facultades de educación y en otras facultades donde el estudiante se forma en profesiones disciplinares ajenas a la educación; pero con el pasar del tiempo, terminan inmersos en el sistema docente del país, sin formación pedagógica en algunos casos. Por ende, esto constituye una carencia a nivel de formación inicial del profesorado y el desarrollo de las competencias básicas, por ello los objetivos que nos planteamos con la realización de este trabajo son los que enunciamos a continuación.

\section{Objetivos}

\subsection{Objetivo General}

- Proponer un modelo de desarrollo de las competencias básicas para la formación inicial del profesorado no universitario.

\subsection{Objetivos Específicos}

- Analizar el estado actual de la formación inicial del profesorado y el desarrollo de competencias básicas, en el proceso de enseñanza - aprendizaje.

- Establecer en el plan de estudio el desarrollo de las competencias básicas en la formación inicial del profesorado.

- Realizar una propuesta con recomendaciones concretas, realistas y coherentes de corto, medio y largo plazo, con el propósito de fortalecer el proceso de formación de las futuras generaciones docentes en el país.

\section{Propuesta de Innovación}

La innovación que aqui se pretende proponer para la formación inicial del profesorado no universitario del país y que parte de la realidad anteriormente expuesta. Consistiría en el desarrollo de las competencias básicas para los futuros docentes y que dicho elemento de innovación se tenga en cuenta en el curriculo de los estudiantes de los diferentes programas de profesorado que se imparten en algunas universidades.

Debido a que ello nos llevaría a tener en el país docentes comprometidos con el entorno y capaces de tomar sus propias decisiones de manera autónoma en relación con la realidad en la que se encuentra inmerso y donde desarrolla su práctica docente.

Además, el trabajar desde la formación inicial el desarrollo de las competencias básicas con los futuros maestros nos podría garantizar en un futuro, tener maestros abiertos a nuevas iniciativas que puedan mejorar el proceso de enseñanza - aprendizaje. Donde no solo los estudiantes aprenden si no que el docente también lo hace, a partir de está experiencia. 
Constituyéndose de esta manera el acto educativo como un proceso abierto, reciproco, actual porque respondería a las realidades del contexto, en otras palabras los maestros más allá de ser especialistas en un área disciplinar, se adueñarían de su práctica educativa y tomarían decisiones responsables, autónomas con profesionalismo acerca de su acción docente (Bellver,2016).

Por tanto, el siguiente articulo es resultado de una revisión bibliográfica realizada, que muestra la situación actual de la formación inicial del profesorado no universitario para Colombia y pretende proponer y dar a conocer la importancia que tiene la formación en competencias básicas para el profesorado no universitario como un elemento de innovación en el país y de está manera aportar a la calidad de la educación Colombiana.

\section{Metodología}

Se realizaron consultas a repositorios como Google Scholar, Scopus, web of science, bases de datos de la universidad pedagógica nacional y algunas bases de datos de acceso libre como: Dialnet plus, Science.gov. Además se consultaron las revistas en educación como la Iberoamericana de educación, revista de educación de España.

Las palabras claves que se utilizaron como criterio de búsqueda fueron: competencias, educación y profesorado principalmente. En el caso de las bases de datos consultadas se tuvo en cuenta como criterio de búsqueda el área a consultar, el tipo de fuente y cuartiles de las revistas encontradas, así como el año de publicación de los trabajos.

Una vez aclarado los criterios de búsqueda, se procedió a realizar la consulta en las diferentes fuentes antes mencionadas. De toda la documentación revisada que fueron aproximadamente 35 articulos, se seleccionaron 19, donde 12 correspondían a estudios en otros países teniendo en cuenta los criterios de búsqueda descritos anteriormente.

Del ámbito internacional 6 de ellos pertenecían a publicaciones españolas y 2 a publicaciones de trabajos hechos en Chile, el resto de publicaciones respondían a estudio realizado: en Ecuador, Argentina, Mexico y Venezuela con una publicación respectivamente y 7 articulos que respondían al ámbito colombiano.

Uno de los criterios que se tuvo en cuenta al momento de seleccionar la documentación fue que los trabajos publicados guardaran relación con el tema. Además, se tuvo en cuenta algunas publicaciones relacionadas con estudios hechos en otros países que han trabajado en esta línea y que algunos son referentes y pioneros, esto con el fin de cotrastar con el estado actual que se tiene del tema en Colombia. Ello permitió generar la reflexión desde experiencias hechas en dichos países.

Como criterios de inclusión y exclusión de los documentos se tuvo en cuenta los trabajos publicados los últimos veinte años, que hablaran de la formación docente en relación con las competencias. Como criterios de exclusión se tuvo en cuenta los artículos que no guardaran relación con el tema, publicaciones que no estuvieran enfocada a la formación del profesorado aunque incluyera el tema de las competencias, estudios que no arrojaran datos 
sobre el estado actual de la formación del profesorado en Colombia y la calidad docente en el país.

Esto permitó obtener una visión general que nos llevó al análisis del estado actual de la formación inicial del profesorado no universitario y el desarrollo de competencias básicas en el país.

\section{Resultados}

De toda la documentación revisada fueron seleccionados un total de 19 articulos donde 12 corresponden a estudios en otros países teniendo en cuenta los criterios descritos anteriormente. Del ámbito internacional 6 de ellos pertenecían a publicaciones españolas y 2 a publicaciones de trabajos hechos en Chile, el resto de publicaciones respondían a estudio realizado en Ecuador, Argentina, Mexico y Venezuela con una publicación respectivamente.

En el caso de los estudios publicados para España, se evidencia que el tema de las competencias es una temática abordada y materia de estudio para el proceso de enseñanza - aprendizaje en el país en todos los niveles educativos. Encontrandose de esta manera que hay trabajos publicados a nivel no solo de formación para rofesores universitarios, sino también para profesores de educación básica.

Algo que llama la atención es que los estudios abordan la parte ética en el campo de la docencia y en esta línea de las competencias también se ve una preocupación por estudiar el perfil del docente no solo universitario sino también de formación básica, lo que permitiría tener una base al momento de abordar estos aspectos en estudios que se realicen a futuro en otros contienentes y en territorio español contar con una herramienta al momento de selección de docentes.

Para el caso de los estudios reportados para américa latina encontramos que para Chile los dos trabajos que hemos tenido en cuenta en este caso apuntan a estudios recientes realizamos dentro del rango que hemos tenido como criterio de inclusión para nuestra investigación y que apuntan al trabajo en la formación inicial del profesorado con base en competencias.

Arrojando en uno de ellos el imperativo de seguir profundizando en esta formación en los años de ejercicio del docente ya que según Ávalos (2004) la formación y especialmente la permanente debe ser en el profesorado algo que se debe ir consolidando con el tiempo ya que está empieza el día que salen de la universidad o centro de formación los nuevos docentes.

Del trabajo publicado por el Instituto de Investigaciones en Ciencias de la Educación en Buenos Aires Argentina en el año 2007, se resalta que este Instituto tiene en cuenta para las nuevas generaciones docentes y para la Universidad en concreto, la importancia de trabajar el desarrollo profesional docente en relación a las realidades actuales de la educación "esta realidad de cambios y nuevos desafios que la escolaridad contemporánea plantea es campo de estudio y trabajo para la educación y formación de nuevos profesores hoy"( 
Instituto de Investigaciones en Ciencias de la Educación, Facultad de Filosofía, Universidad de Buenos Aires, 2007).

Para el caso de México se encontró que el estudio relacionado con el tema aborda la formación basada en competencias en educación superior y esté va de la mano con el estudio de un perfil ético que lleve a la transformación del proceso formativo del futuro profesional en educación. Proponiendo para este caso el aprendizaje basado en problemas y apoyado a través de talleres que permitan desarrollar las competencias desde las aulas de clases.

En el estudio que hemos encontrado reportado para Venezuela se abordó el estado del arte de las competencias del docente en la sociedad de la globalización. Es así, como en el estudio se pretende ofrecer herramientas de fortalecimiento y/o desarrollo de las competencias del siglo XXI que debe tener el docente para responder a las realidades actuales.

En el ámbito nacional colombiano hemos tenido en cuenta 7 articulos que después de haberlos estudiado teniendo en cuenta los criterios que se han abordado en la revisión bibliográfica nos permite aterrizar la temática a la realidad local y tener un espectro general del estado actual de la formación en competencias para docente no universitarios en el país.

Es así, como Calvo., et al, 2004 señala un diagnóstico de la formación docente en el país. Este estudio tuvo en cuenta las escuelas normales superiores y las facultades de educación de las universidades del país. Aunque se mostró avances a nivel de formación del profesorado persiste según el autor una estructura a nivel de currículo que no responde a la realidad de la formación hoy.

Por su parte Camargo y Pardo (2008) reportan un estudio en competencias de profesores de pregrado, haciendo énfasis en la importante formación del profesorado universitario y apartir de un diseño y validación de un instrumento de evaluación poder plantear una evaluación docente basada en competencias.

En tanto que, Parra., et al 2010 publica un estudio de formación de los profesores universitario en relación a las competencias, concluyendo que la formación de los docentes es una tarea todavía pendiente en el país.

Cabra y Marín (2014) señalan algunos resultados que obtuvieron de un estudio sobre formación inicial del profesorado en el país desde áreas muy especificas como: sociales, Artes y Humanidades, Ciencias Naturales y Educación - Pedagogía mostrando avances en la formación del profesorado en competencias. De esta manera se deja ver que desde el 2010 al 2014 se muestra un avance en cuanto a la formación del profesorado que responda a las situaciones que exige la educación hoy.

Si detallamos los estudios mas recientes con respecto al tema tenemos que Jurado (2016) publica el trabajo hacia la renovación de la formación de los docentes en Colombia, donde concluye que si bien es verdad que se ha avanzado en materia de formación docente el origen del problema en el país a nivel de formación del profesorado responde a situaciones estructurales debido a las fisuras que presenta el sistema de educación del país. 
Bautista y Gómez (2017) expresan que las escuelas normales en el país no son instituciones de formación de alto nivel académico para docentes del nivel primario de la educación. Sumado a ello las facultades de educación de las universidades están reproduciendo el modelo propuesto por las escuelas normales para la formación docente esto podría estar truncando un avance significativo en cuanto a formación del profesorado e influyendo a su vez en la calidad educativa colombiana.

Además, se encontró en el estudio realizado por Cabeza y Lombana (2018) que en el país la profesión docente para las nuevas generaciones no es opción a tener en cuenta al momento de estudiar y profesionalizarse en ella ya que están influyendo factores económicos laborales y sociales en dicha postura de las generaciones actuales.

Desde este espectro del estado actual que se tiene en la formación inicial de profesorado no universitario se plantea el desarrollo de las competencias básicas para los futuros educadores. El cual debería pasar del énfasis en conocimientos conceptuales y factuales al enfoque en el desempeño integral ante actividades y problemas.

De esta manera al momento que el futuro docente este desarrollando su labor como maestro podrá concebir su ejercicio como algo integral que implica la articulación del conocer con el plano del hacer y del ser.

Así, la educación que se imparte podrá ser llevada al contexto local, regional e internacional y esto permitiría contrastar lo que en el país se lleva a cabo con otras experiencias educativas en cuanto a la formación inicial del profesorado que se realizan en otros países.

Siempre buscando mejorar dicho proceso de formación y así los estudiantes de los programas de formación docente junto con quienes coordinan las facultades de educación estarían en constante búsqueda, selección, comprensión, sistematización critica, creación de nuevas expeiencias y los docentes que imparten los diversos cursos actualizarían el currículo y la aplicación de contenidos a las realidades concretas.

Por tanto, tendríamos que la formación basada en competencias implicarìa que el aprendizaje comience a ser el centro de la educación, más que la enseñanza. Esto significa que en vez de centrar en las facultades toda la atención y energías para que aprendan nuestros estudiantes a cómo dar una clase y preparar los recursos didácticos para ello, nos enfocaríamos en el reto de establecer con qué aprendizajes vienen nuestros futuros docentes, y de allí mirar cuáles serían sus expectativas, debilidades, carencias para desde allí empezar a construir juntos el conocimiento que responda a las necesidades de los contextos de donde vienen.

Finalmente, lo que buscamos con está innovación educativa en la formación inicial del profesorado no universitario es que se puedan desarrollar las competencias básicas como las que señala González y Wagenaar (2003) Capacidad de análisis, capacidad de organizar y planificar, conocimientos generales básicos del área a impartir, conocimientos básicos de la profesión, comunicación oral y escrita en la propia lengua, habilidades de gestión de la información, resolución de problemas, toma de decisiones, trabajo en equipo, habilidades 
interpersonales. Todo ello apuntaría a la calidad de la educación que se espera en el país para los próximos años.

\section{Conclusiones}

Ante los resultados anteriormente expuestos, se puede ver que Colombia cuenta con una estructura de formación docente que responde a épocas pasadas, ello se evidencia en las publicaciones que hemos estudiado.

Además, es de vital importancia resaltar que si nos vamos a la ley general de educación con la que cuenta el país esta es del año 1994 y aunque da aportes muy significativos algunos no responden a las situaciones educativas actuales, quizá porque está fue escrita en tiempos pasados. Por tanto, se podría decir que el ministerio de educación colombiano debería revisar dicha ley de educación y hacer los ajustes necesarios para responder eficientemente a las necesidades educativas en cuanto a la formación del profesorado.

Se evidenció que en el país existen tres vías de profesionalización en la formación inicial docente: la formación de carácter compelementario, la formación de pregrado mediante licenciatura y la formación de pregrado en otros programas universitarios y habrá que mirar a nivel de formación para el profesorado en el país que opciones son pertinentes para aportar significativa y asertivamente a las necesidades que se tenga. Para que de esta manera la calidad de la educación vaya mejorando en algunos aspectos desde la formación de los profesionales en la educación.

Por todo ello, la investigación que hemos realizado aporta datos que sirven como base para estructurar futuras investigaciones en el tema, con el fin de buscar estrategias y planes formativos que permita por una parte cualificar al profesorado en el país, no solo a nivel de docentes universitarios sino también a nivel de docentes no universitarios ya que es en la educación básica donde sentamos las bases para la formación futura de generaciones.

Por ello la propuesta que ahora se hace va en la línea de incluir en los curriculos de formación inicial del profesorado no universitario el desarrollo de competencias básicas para que los futuros docentes sean capaces de dar respuesta a los desafíos que la educación actual nos plantea y esto nos permitiría mejorar la calidad de la educación en el país.

Finalmente, el gobierno deberá analizar y reflexionar las políticas en cuanto a factores económicos y laborales para la profesionalización docente ya que esto esta influyendo a que las nuevas generaciones no vean la docencia como camino de realización personal y profesional lo que a mediano y largo plazo podría constituir un problema para aquellas zonas donde la presencia de docentes es escasa y necesaria.

\section{Referencias}

Amor, M. (2018). Las Competencias Generales en la Formación inicial del Profesorado, un Estudio Comparativo entre Estudiantes, Docentes y Graduados de los Títulos Universitarios de Educación. Revista de la Facultad de Educación UNED Educación XXI, 1 (1), 239 -261 
Bautista, M. y Gómez, V. (2017). Calidad Docente: un Desafio para la Tradición Pedagógica en Colombia. Medellín (Colombia). Universidad Nacional de Colombia, Colección General, Biblioteca Abierta.

Bellver, C. La formación del profesorado en Competencias. Democracia y Educación en la formación docente, Valencia, Universitat de Vic, Universitat de Vic - Universitat Central de Catalunya. 2016, Pág. 87 -91.

Bozú, Z. y Canto Herrera, P. (2009) El Profesorado Universitario en la Sociedad del Conocimiento: Competencias Profesionales Docentes. Revista de Formación e Innovación Educativa Universitaria, 2 (2), 87-97.

Bustos, R. (2015). El Desarrollo de Competencias Genéricas en Estudiantes que se Desempeñan como Tutores Pares en la Universidad de Tarapacá. Revista Colombiana de Educación, 2 (75), 99 -117.

Cabeza, L. Zapata, A. y Lombana, J. (2018) Crisis de la Profesión Docente en Colombia: Percepciones de Aspirantes a otras Profesiones. Revista Educación y Educadores, 21 (1), 5172

Cabra Torres, F. Marín Díaz, D. (2014) Formar Para Investigar e Innovar: Tensiones y Preguntas Sobre la Formación Inicial de Maestros en Colombia. Revista Colombiana de Educación, 1 (68), 149 -171

Calvo, G. Rendón Lara, D. Rojas García, L. (2004) Un Diagnóstico de la Formación Docente en Colombia. Revista red académica Universidad Pedagógica Nacional, 2 (47), 30-37

Camargo Escobar, I. Pardo Adames, C. (2008) Competencias Docentes de Profesores de Pregrado: Diseño y Validación de un Instrumento de Evaluación. Revista Universidad Católica de Colombia, 7 (2), 441 -455

Davidson Ávalos, B. (2004) Renovando la Formación Docente Inicial. Algunas Consideraciones. Revista Red Académica Universidad Pedagógica Nacional, 2 (47), 1 -15

El Instituto de Investigaciones en Ciencias de la Educación, Facultad de Filosofía, Universidad de Buenos Aires (2007). La Formación y el Desarrollo Profesional Docente Frente a los Nuevos Desafíos de la Escolaridad. Revista de Currículum y Formación de Profesorado, 11(1), p. 0

Escobar Roldán Mariana (2017) El país donde no faltan maestros. Nota Periodística de EL ESPECTADOR tomado de https://www.elespectador.com/noticias/educacion/el-paisdonde-no-faltan-maestros-articulo-450612. 
Estado Actual de la Formación Inicial en Competencias Básicas del Profesorado no Universitario de Colombia

Gairín, Joaquín (2011) Revisión Sobre Algunos Desarrollos Controvertidos de la Formación por Competencias, para Delimitar de Manera Específica Funciones, Perfiles y Competencias de los Programas de Formación del Profesorado. Revista Bordón, 63 (1), 93-108

Jurado Valencia, F. (2016) Hacia la Renovación de la Formación de los Docentes en Colombia: Ruta Tradicional y Ruta Polivalente. Revista Pedagogía y Saberes Universidad Pedagógica Nacional, 1 (45), 11-22

Ley general de educación Colombia Ley 115 de 1994 recuperado de https://www.mineducacion.gov.co/1621/articles-85906_archivo_pdf.pdf (Consultado en diciembre de 2018).

López, M. León, M. y Pérez, P. (2018) El Enfoque por Competencias en el Contexto Universitario Español. La Visión del Profesorado. Revista RIE, 2 (36), 529 -545

Mendoza Lira M. (2013) Adquisición y Desarrollo de Competencias Profesionales en el Prácticum de los Grados de Magisterio: Estudio Empírico desde la Perspectiva de los Estudiantes. Tesis doctoral. Universidad Complutense de Madrid.

Muñoz, G. Rodríguez, P. y Luque, M. (2007) La Formación Inicial del Profesorado de Educación Secundaria en España: Perfil y Motivaciones del Futuro Docente. Revista de la Facultad de Educación UNED -Educación XXI, 1 (1), 71-92

Parra Moreno, C. Ecima Sánchez, I. Gómez Becerra, M. y Almenárez Moreno, F. (2010) La Formación de los Profesores Universitarios: Una Asignatura Pendiente de la Universidad Colombiana. Revista Educación y Educadores, 13(3), 421-452

Pilonieta G. (2014) La Formación del Maestro. Magisterio.com.co (Revista electrónica) Recuperado de https://www.magisterio.com.co/articulo/la-formacion-del-maestro (consultado de diciembre de 2018).

Restrepo Aguirre, J. y Gámez Navío A. (2016) Las Competencias del Docente de Maestría en Universidades Colombianas: Apreciaciones de Alumnos y Profesores. Revista Civilizar Ciencias Sociales y Humanas, 16 (31), 191-204.

Tobón S. (2008) La Formación Basada en Competencias en la Educación Superior. Revista de Dirección de Investigaciones y Postgrado Universidad Nacional Abierta de México, grupo Cife, 10 (2), 6 - 30 .

Toro Álvarez, R. (2010) El Estado del Arte de las Competencias del Docente: En la Sociedad de la Globalización, Una Mirada en el Escenario Venezolano. Revista EduTicInnova,1(2), 56- 69

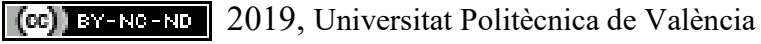


Torres, R. (2004) En Nuevo Rol Docente: ¿Qué Modelo de Formación, Para Qué Modelo Educativo? Revista Red Académica Universidad Pedagógica Nacional,2(47), 19-38

Zabalza, M. (2009) Ser Profesor Universitario Hoy. Revista La Cuestión Universitaria, 1 (5), $68-80$ 